Haïti : I'oraison démocratique

\title{
Débats identitaires et impasse statutaire à Porto
} Rico

Justin Daniel

\section{(2) OpenEdition}

12 Journals

Édition électronique

URL : http://journals.openedition.org/plc/770

DOI : $10.4000 /$ plc. 770

ISSN : 2117-5209

Éditeur

L'Harmattan

\section{Édition imprimée}

Date de publication : 1 janvier 1998

Pagination : 323-341

ISSN : 1279-8657

\section{Référence électronique}

Justin Daniel, « Débats identitaires et impasse statutaire à Porto Rico », Pouvoirs dans la Caraibe [En ligne], 10 | 1998, mis en ligne le 02 mai 2011, consulté le 22 septembre 2020. URL : http:// journals.openedition.org/plc/770 ; DOI : https://doi.org/10.4000/plc.770

Ce document a été généré automatiquement le 22 septembre 2020.

(c) Pouvoirs dans la Caraïbe 


\title{
Débats identitaires et impasse statutaire à Porto Rico
}

\author{
Justin Daniel
}

\section{RÉFÉRENCE}

NANCY MORRIS, Puerto Rico, Culture, Politics and Identity. Westport : Praeger, 1995.

FRANCES NEGRÓN-MUNTANER, RAMÓN GROSFOGUEL (eds), Puerto Rican Jam, Essays on

Culture and Politics. Minneapolis : University of Minnesota Press, 1997.

JOSE LUIS MENDEZ, Entre el limbo y el consenso, El dilema de Puerto Rico para el próximo siglo. San Juan : Ediciones Milenio, 1997.

1 L'approche du centenaire de l'annexion de Porto Rico par les Etats-Unis a incontestablement contribué à relancer la réflexion non seulement sur les problèmes d'identité et de culture, mais aussi sur l'avenir politico-institutionnel de l'ancienne colonie espagnole. S'il est vrai que ces questionnements n'ont jamais abandonné la scène intellectuelle et politique insulaire, il est non moins sûr qu'ils connaissent depuis quelques année un regain de faveur : plus que jamais persiste à Porto Rico le sentiment d'un malaise profond dans les rapports avec les Etats-Unis; sentiment nourri sans doute par l'échec des tentatives visant à transcender un statut politique qui semble avoir épuisé ses potentialités et sa capacité à réaliser une articulation satisfaisante entre les trois "libertés" fondamentales - économique, politique et culturelle identifiées par son père fondateur dans les années 40. D'échecs relatifs en tentatives avortées, l'avenir politique de Porto Rico semble se dessiner en creux, sous la forme d'une impasse statutaire qui, par un effet de retour, focalise l'attention des chercheurs, dont la réflexion est rarement dissociable de préoccupations directement politiques, sur des thèmes - le nationalisme, l'identité, la culture et le politique... - considérés comme autant de passages obligés pour rendre intelligible une situation relativement complexe. Il faut dire que la société portoricaine, dans sa (vaine?) tentative pour 
concilier le "meilleur des deux mondes" offre un champ d'observation pour le moins intéressant à propos de ces questions.

2 Les trois ouvrages analysés ci-dessous n'ont pas la même ambition : certains d'entre eux privilégient la rigueur du discours universitaire là ou les autres épousent la liberté de ton qu'autorisent les essais. Ils ont néanmoins le mérite de contribuer à des débats, certes récurrents, mais abordés en des termes renouvelés en bousculant parfois certaines idées reçues.

3 A commencer tout d'abord par l'ouvrage de Nancy Morris, Puerto Rico, Culture, Politics and Identity. Spécialiste des problèmes de communication, d'origine américaine tout en ayant longtemps travaillé sur les sociétés d'Amérique du Sud avant de gagner l'Ecosse, l'auteur se penche ici sur la question de l'identité nationale à Porto Rico à partir de deux sources de données: d'une part, des documents historiques de première et de seconde main ; d'autre part, une enquête de terrain qui l'a conduit à interroger, sous forme d'entretiens, dix-neuf hommes politiques représentatifs des trois principaux partis portoricains et à organiser onze entretiens au sein de groupes composés de militants issus également des trois mouvements qui structurent la vie politique insulaire. La richesse des entretiens témoigne d'une excellente maîtrise de cette technique d'investigation qui, il faut le rappeler, est d'une manipulation délicate, a fortiori dans un contexte caractérisé par des débats linguistiques passionnés et dans lequel l'usage d'une langue est rarement perçu comme neutre. On peut regretter que l'approche strictement élitiste retenue par l'auteur l'ait amené à renoncer à toute extension d'une technique d'investigation riche de potentialités au delà des cercles politiques officiels. Car l'ouvrage repose sur une hypothèse clairement annoncée : les leaders politiques jouent un rôle décisif dans les phénomènes de construction identitaire, non seulement en ayant accès aux canaux permettant de façonner l'identité elle même, mais aussi en étant sensibles, dans leur quête de soutiens politiques, aux attitudes des électeurs face aux enjeux identitaires. Dès lors, il faut bien admettre que Nancy Morris explore essentiellement les stratégies et les représentations identitaires des élites politiques au risque de négliger la part prise par tous les autres acteurs dans la construction d'une identité dont elle souligne, très justement par ailleurs, le caractère multiple.

4 L'ouvrage se décompose en trois parties précédées d'un chapitre théorique à travers lequel l'auteur s'efforce de définir les principaux concepts servant de support à son analyse. S'inspirant très largement des travaux d'Eric Hobsbawn, elle définit la nation comme "une communauté de personnes qui se définissent comme telle [et] qui partagent un sentiment de solidarité fondé sur la croyance en un héritage commun et qui revendiquent des droits pouvant inclure l'autodétermination " (p. 12) et l'identité nationale comme "le sentiment individuel d'appartenir à une collectivité qui s'autoproclame une nation " (p. 14). Sur la base de ces définitions, Porto Rico apparaît sans l'ombre d'un doute comme une nation qui présente la particularité de ne pas être érigée en Etat. Le terme "nation" ou "nationalité" est d'ailleurs mobilisé par la majorité des hommes politiques insulaires (huit sur quatorze) dans la définition qu'ils proposent de Porto Rico et l'existence d'une culture et d'une identité portoricaines est reprise en chœur par les adhérents de l'ensemble des partis, indépendamment de leur option sur le plan politico-institutionnel. Le paradoxe est donc frappant entre la faiblesse des soutiens électoraux dont bénéficie l'indépendance et la force avec laquelle est affirmée, selon des modalités et des voies variables, une identité distincte. 
5 La première partie de l'ouvrage correspond à la description de la période qui s'étend de 1898, date de l'annexion de l'île par les Etats-Unis à 1993, année durant laquelle fut organisé le dernier référendum à ce jour sur la question du statut. Il s'agit en réalité de près d'un siècle de relations complexes et parfois mouvementées entre l'ancienne colonie espagnole et sa nouvelle métropole. Nancy Morris rappelle ainsi les hésitations qui ont accompagné la mise en place d'un gouvernement civil, l'octroi de la citoyenneté américaine en 1917, le processus d'“ américanisation" et la montée d'une insatisfaction et de frustrations qui s'expriment dans les années 30 à travers les canaux intellectuels et politiques. En 1938 se produit un événement dont les répercussions ultérieures sur la vie politique insulaire sont considérables: l'aile du Parti libéral favorable à l'indépendance fait scission et donne naissance au Parti Populaire Démocratique (PPD) à l'initiative de Luis Muñoz Marín, grande figure du paysage politique insulaire, qui choisit comme symbole le jíbaro dans le souci manifeste d'incorporer le monde rural dans sa stratégie. Cette évolution annonce la mise en place du statut d'Etat Libre Associé intervenu en 1952, et qui suscitera plus tard de nouvelles contestations et interrogations sur l'avenir. Elaborée principalement à partir de sources secondaires, cette partie de l'ouvrage a néanmoins le mérite d'attirer l'attention sur les différents symboles mobilisés - le drapeau, l'hymne, les jours fériés - dans le cadre de la construction d'une identité, voire d'un nationalisme culturel, transcendant dans une large mesure les divergences politiques.

C'est là d'ailleurs un constat que vient conforter la deuxième partie de l'ouvrage. Ici l'auteur s'attache, sur la base des entretiens réalisés, à mettre au jour les attitudes des leaders politiques et des adhérents des partis face à la question de l'identité portoricaine. Nul doute en effet que cette dernière est affirmée avec force d'un bout à l'autre du spectre politique et que les Portoricains, indépendamment de tout clivage politique, s'accordent sur ce qui, à leurs yeux, en constitue les éléments-clés et tendent à les distinguer des autres: la langue, l'histoire et les traditions, la musique, les coutumes alimentaires... Il est à noter l'importance accordée à la participation des sportifs portoricains, sous leur propre bannière, aux jeux olympiques, et de manière plus générale, aux compétitions internationales. Largement couverts par les médias locaux, ces événements permettent de bénéficier d'une représentation distincte et de se projeter en tant que telle sur la scène internationale, non sans une certaine ambiguïté : si les partisans du statut $\mathrm{d}^{\prime} \mathrm{ELA}^{1}$ sont unanimes à faire référence à l'équipe olympique, il n'en va de même pour les protagonistes de l'indépendance ou de l'annexion soucieux, semble-t-il, de se démarquer de tout ce qui pourrait cautionner la représentation officielle de l'ELA (p. 93).

7 La grande majorité des enquêtés, y compris ceux qui se situent dans la mouvance annexionniste s'identifient avant tout comme portoricains (chapitre 5). Les réponses apportées par ces derniers vont du rejet de toute identification aux Etats-Unis à l'affirmation, sur un mode égalitaire, d'une double appartenance, américaine et portoricaine. Le sentiment d'appartenir à la Caraïbe - et à l'Amérique latine - varie pour sa part en fonction d'une combinaison complexe de facteurs faisant intervenir les préférences politiques, le lieu de résidence dans l'île et l'âge. Ainsi les partisans de l'autonomie et de l'indépendance ont-ils tendance à privilégier leurs affinités avec la Caraïbe et l'Amérique latine au détriment des Etats-Unis. On retrouve des divergences semblables à propos des allégeances locales, des Nuyoricans ${ }^{2}$, de sorte que la catégorie centrale autour de laquelle se construit l'identité reste incontestablement le fait 
portoricain sur lequel viennent se greffer d'autres appartenances revendiquées avec plus ou moins de force. D'où les deux caractéristiques de l'identité portoricaine isolées par l'auteur : fluidité et cristallisation sur la base des différentes expériences sociales.

La capacité de résistance (resilience) de l'identité culturelle dans le contexte de la domination américaine donne lieu à des appréciations divergentes dans la troisième partie de l'ouvrage. Si les enquêtés sont unanimes à reconnaître l'influence des EtatsUnis, ils évaluent sa portée en fonction de leur affiliation partisane : là où les tenants de l'annexion soulignent les éléments positifs de la présence américaine ainsi que la capacité de résistance de l'identité, y compris en cas de transformation de l'île en $51^{\text {ème }}$ Etat, les partisans de l'autonomie et de l'indépendance déplorent les conséquence néfastes d'une telle présence, tout en reconnaissant qu'elle a permis d'assurer le fonctionnement de la démocratie ; là où les uns restent confiants, les autres redoutent une assimilation culturelle. Il reste que la plupart des enquêtés ne considèrent pas comme une réelle menace certains produits culturels importés, qu'il s'agisse de la musique nord-américaine et, de la cuisine rapide qui connaissent un important succès et coexistent avec des habitudes locales. A l'inverse, d'autres produits, pratiques, ou symboles font l'objet d'un incontestable rejet comme, par exemple, la fête de Santa Claus qui tend à supplanter la fête locale des "Rois mages ", la télévision par câble, ou encore la langue anglaise. Pour l'auteur, la perception d'une menace pesant sur les symboles de l'identité a, par un effet de retour, contribué à renforcer ces derniers. Nancy Morris souligne ainsi la capacité de la culture portoricaine à absorber et à adapter les éléments importés, tout en se préservant et en se renouvelant.

9 Le dernier chapitre est d'ailleurs consacré à la résistance de la culture locale. Il faut dire que les leaders politiques, quelles que soient leurs divergences sur la question du statut, s'accordent tous sur la défense de cette culture et le respect de l'identité nationale. Cette identité nationale s'exprime à travers un corpus symbolique qui est loin d'être figé. Trois éléments constitutifs de l'identité ont ainsi changé de sens à travers l'histoire : dissociée de l'annexion, la citoyenneté américaine a été d'abord rejetée par la majorité du Parti Unioniste en 1916, avant d'être acceptée l'année suivante comme un élément du dispositif de décolonisation, d'être fixée dans la constitution de 1952 et d'être revendiquée aujourd'hui par la majorité de la population, y compris une frange du mouvement indépendantiste ${ }^{3}$; la participation des Portoricains aux compétitions sportives internationales génère des tensions avec les Etats-Unis qui traduisent assez bien la difficile conciliation entre les avantages inhérents à la citoyenneté américaine et l'adhésion passionnée à l'idée de "sports citizenship" (citoyenneté sportive), une notion dérivée des règles permettant aux Portoricains de représenter leur île (et non l'État dont ils sont les citoyens) et qui joue un rôle essentiel dans la construction identitaire quoique inconnue avant le milieu de ce siècle ; enfin, le drapeau portoricain fut conçu initialement comme un symbole de la lutte pour l'indépendance durant la domination espagnole, avant de rallier l'ensemble de la population, indépendamment des préférences en matière de statut, d'être adopté en 1952 pour représenter le Commonwealth ${ }^{4}$ et de flotter aux côtés de la bannière étoilée comme emblème d'une relation hétérodoxe et délicate à assumer (p. 159).

10 Si les emblèmes et les symboles qui servent de support à l'identité ne sont pas pérennes, ils font également l'objet d'une appropriation différenciée de la part des groupes sociaux. Ainsi, la langue espagnole, érigée en symbole d'identification par rapport à l'anglais, sert-elle en même temps de marqueur à différents groupes 
énonçant leurs positions divergentes et concurrentielles au sein de l'espace public sur les questions d'identité culturelle et de statut politique. De manière plus générale, chacune des représentations identitaires est constamment négociée par une très grande diversité de groupes, de sous-groupes et d'acteurs sociaux.

11 Au total l'ouvrage de Nancy Morris présente un triple intérêt: d'abord, nourri par une recherche sur le terrain (on ne soulignera jamais assez la richesse des entretiens), il permet de mettre au jour les attitudes des leaders, mais aussi des adhérents des trois principaux partis politiques sur une question - l'identité - qui n'a jamais cessé de tramer la vie politique insulaire, en particulier depuis l'arrivée des Américains. En second lieu, il constitue un apport indéniable sur le plan théorique. Refusant toute conception substantialiste de l'identité, l'auteur déjoue en même temps le piège clairement mis en évidence par Christine Chivallon ${ }^{5}$ - d'identités mobiles exclusives des expériences sociales dans lesquelles pourtant elles s'enracinent et se recomposent. Enfin, Nancy Morris a le mérite de rappeler la dimension stratégique des phénomènes de construction identitaire indissociables des enjeux du moment et des modalités d'appropriation de ces enjeux par des acteurs en lutte pour le pouvoir et/ou la reconnaissance symbolique.

12 Malgré tout, l'ouvrage n'échappe pas à un reproche majeur. Autant, les enquêtes réalisées sur le terrain se sont révélées d'une incontestable richesse, autant on ne peut s'empêcher de regretter que l'auteur ait choisi de les limiter aux élites politiques et à une frange de la jeunesse au nom d'un postulat plus ou moins contestable: les élites sont nécessairement sensibles aux attitudes de l'électorat au regard de la question identitaire et reprennent à leur compte ses conceptions et attentes. Il en résulte que les conclusions de l'auteur peuvent être difficilement généralisées à l'ensemble de la population: d'une part, elles concernent, par un effet de la méthode utilisée, des leaders politiques relativement jeunes, au risque de procéder, par delà des clivages partisans, à une homogénéisation quelque peu déformatrice ; d'autre part, les données recueillies se réfèrent aux attitudes des leaders politiques, en négligeant les secteurs populaires, appréhendant du même coup la question de l'identité exclusivement à travers le moule - pour ne pas dire le prisme déformant - de l'élite politique et des affiliations partisanes. Or il est à peine besoin de rappeler que les acteurs politiques sont loin d'être les seuls à participer aux processus de construction identitaire et le rôle joué en la matière par les secteurs populaires.

13 Telle est d'ailleurs l'impression qui se dégage de la lecture de Puerto Rican Jam. Rédigé par une nouvelle génération d'intellectuels portoricains qui entendent proposer une analyse originale du colonialisme et des discours nationalistes, cet ouvrage comprend une douzaine d'articles dont le ciment est en quelque sorte constitué par une approche très largement sensible au postmodernisme ambiant. Les auteurs ambitionnent de sortir de la dichotomie classique opposant le " mal " (le colonialisme) à son " remède " supposé, (le nationalisme), en produisant un discours novateur permettant d'aborder les défis auxquels se trouvent confrontés les Portoricains en cette fin de siècle. Dans une longue introduction, Ramón Grosfoguel, Frances Negrón-Muntaner et Chloé Georas, proposent non pas de régler, comme beaucoup d'autres dans le passé, la question du colonialisme à Porto Rico, mais de jeter un regard critique sur le couple discursif colonial/national à partir de la mise au jour d'un ensemble de stratégies trop souvent négligées par les analyses classiques. Par une sorte de clin d'œil - non clairement explicité - à la théorie critique chère à Habermas, ils affirment sans 
ambiguïté que leurs théories sont constitutives de leur action politique et que le lien entre les deux doit être examiné de manière critique.

Analysant les discours sur le colonialisme et le nationalisme, les auteurs soulignent qu'ils ont entrés en crise et que leur capacité de mobilisation s'est considérablement affaiblie. Sans doute faut-il y voir la résultante d'une trajectoire historique singulière que l'on peut difficilement restituer à partir de l'opposition simpliste entre les tenants du colonialisme et ceux qui le combattent : les groupes sociaux subordonnés ont dirigé leur lutte non pas tant contre la métropole que contre les élites politiques locales, en mobilisant les discours sur les droits de l'homme produits par le centre...métropolitain. Mariano Negrón-Portillo ${ }^{6}$ voit dans le mécontentement de la plupart de ces groupes à l'égard de l'élite créole et du régime colonial espagnol l'une des causes principales de l'absence de cohésion du discours nationaliste au début du siècle : de larges secteurs sociaux en voie de prolétarisation ont en effet articulé un projet politique de modernisation dans le cadre d'une alliance avec les Etats-Unis et en opposition à leur ennemi de classe, l'élite créole; inversement, l'orientation hispanisante du discours nationaliste a été source de ressentiment à l'égard non seulement du régime espagnol, mais aussi de ses alliés et de ceux qui prétendent incarner une identité fondée sur l'héritage espagnol. Autrement dit, le clivage central ne se situe pas entre les colonisés et les colons, mais se décompose en de multiples clivages se cristallisant autour des intérêts liés aux appartenances de classe, de race et de sexe qui ne sauraient être regardés comme déterminés par la question nationale. Bien au contraire, ces clivages s'opèrent sur la base d'alliances parfois surprenantes comme le révèle l'analyse - pour le moins provocatrice - proposée par Gladys Jiménez-Muñoz ${ }^{7}$ : au début du siècle, les suffragettes portoricaines ont recherché une alliance avec les féministes américaines qui ont fait appel en leur nom aux membres du Congrès, sans distinction de sexe, pour qu'ils favorisent l'extension du droit de vote aux femmes insulaires dans le cadre des élections locales; en d'autres termes, une stratégie (délibérément tournée contre les hommes portoricains) s'est appuyée sur une solidarité entre femmes (portoricaines et américaines d'origine blanche) issues des classes moyennes et sur une demande d'intervention adressée au centre colonial.

On pourrait multiplier les exemples du même ordre. Ainsi Ramón Grosfoguel ${ }^{8}$ s'efforce $^{\prime}$ de montrer comment le jeu complexe de la politique coloniale américaine ne se résume pas en la poursuite d'intérêts strictement économiques. Il arrive même que plusieurs objectifs contradictoires soient envisagés simultanément dans certaines conjonctures historiques. Le processus d'accumulation capitaliste, souvent présenté comme le moteur de la politique coloniale, a dû s'accommoder de l'extension des droits sociaux et des transferts fédéraux au cours de la seconde moitié du vingtième siècle. Cette évolution a directement affecté la situation des firmes nord-américaines implantées dans l'île, révélant du même coup la dimension symbolique de la présence des EtatsUnis ; ces derniers entendant faire de Porto Rico une sorte de vitrine dans la Caraïbe dans le cadre de leur stratégie globale de développement. De sorte que l'oscillation permanente de la politique coloniale entre des tendances ou des prétentions contradictoires constitue un obstacle à l'émergence d'un nationalisme structuré.

Plusieurs articles présentent, par ailleurs, un point de vue critique sur le statut d'Etat Libre Associé, source de nombreuses illusions. Pour Jaime Benson-Arias ${ }^{9}$ par exemple, Porto Rico demeure une colonie, au sens politique du terme, dans la mesure où ce territoire a été complètement absorbé par l'économie américaine au point d'apparaître 
comme une simple extension régionale de cette dernière tout en lui étant subordonné. Pourtant, l'ELA est sous-tendu par une représentation de l'île en termes de "nation " qui, sans avoir pu s'ériger en Etat, contrôle un espace interne échappant à l'emprise du capitalisme mondial. Le statut d'Etat Libre Associé occulte le rapport colonial unissant Porto Rico aux Etats-Unis et explique d'une certaine façon les tenants et les aboutissants du débat culturel. A n'en pas douter en effet, l'ambiguïté du statut politique ne s'est nullement traduite par une remise en cause de l'identité culturelle, contrairement aux allégations du discours dénonçant "l'impérialisme culturel ", mais au contraire par la démultiplication des niveaux d'identification et par des phénomènes d'hybridation repérables à travers le jeu des acteurs sociaux. Contestant le point de vue négatif des "théoriciens" de l'impérialisme culturel, Raquel Rivera ${ }^{10}$ s'efforce de montrer comment la musique et la culture rap sont le lieu d'expression d'une jeunesse urbaine, noire et démunie permettant en outre de recomposer des solidarités - sur la base de critères de classe et de race - transcendant l'opposition colonial/national. L'essai signale en outre la persistance d'un racisme à l'encontre des noirs à Porto Rico que les discours nationaliste et colonialiste ignore en subsumant dans un prétendu rapport égalitaire les différentes composantes raciales et ethniques de la population.

$\mathrm{Au}$ delà de la question culturelle, plusieurs thèmes sont abordés qu'il serait assurément fastidieux de passer en revue. On se contentera de relever quelques-uns d'entre eux et surtout d'attirer l'attention sur les questions qui sont ainsi soulevées. De ce point de vue, il est clair que Porto Rico est "imaginé" non seulement comme un espace géographique spécifique, mais aussi comme une communauté symbolique revendiquée aussi bien par les membres qui vivent sur l'île que par ceux résidant aux Etats-Unis ${ }^{11}$, avec tout ce que cela suppose comme problèmes. Cette appartenance à "deux mondes " explique l'acuité du débat linguistique ${ }^{12}$ et la difficulté à analyser les représentations collectives que les Portoricains ont forgé d'eux mêmes. Dans l'introduction précitée, les auteurs en viennent à conceptualiser ces représentations à partir d'un double constat dont les éléments sont solidaires : une "ethno-nation " en voie de déterritorialisation aux Etats-Unis et une " ethno-nation " territorialisée à Porto Rico (p. 19).

18 C'est dire que la solution politique à une telle situation n'est pas aisée. Fidèles à leur objectif, sortir du débat actuel en forme de dilemme, et à la démarche annoncée, nourrir leur stratégie politique de leurs analyses scientifiques, Ramón Grosfoguel, Frances Negrón-Muntaner et Chloé Georas considèrent que le projet de décolonisation de Porto Rico ne peut être compris uniquement comme un processus formel d'autodétermination politique, mais doit être envisagé comme une transformation radicale des vieilles hiérarchies coloniales, raciales, sexuelles et de classes forgées dans le cadre d'un rapport de subordination constamment réactivé. Cet objectif suppose la capitalisation des pratiques culturelles et politiques nouvelles, des nouveaux mouvements sociaux, de discours novateurs prenant le contre-pied des prémisses de la pensée politique et des pratiques nationalistes, y compris celles en cours dans la métropole. Pour déconstruire le dualisme improductif colonialisme/nationalisme et dégager de nouvelles perspectives, il convient de recourir à des stratégies dites de “complicité subversive" (Grosfoguel) qui dans le passé ont permis d'arracher des concessions au système colonial tout en anticipant le postmodernisme actuel: le "mimétisme sans identification" ou la "parodie postmoderniste". Les auteurs affirment ainsi qu'une féminisation de la vie politique portoricaine, entendue comme une stratégie non-essentialiste étendant et généralisant aux luttes politiques les comportements féminins - tels que la séduction, l'ambiguïté et la négociation - 
historiquement associés au patriarcat pourrait prouver toute son efficacité dans un contexte de domination. En réalité, plutôt que de se situer en dehors du système capitaliste ou de chercher à l'affronter ouvertement, il convient de le subvertir de l'intérieur par une sorte de complicité active, en le parodiant et en essayant d'en tirer le maximum d'avantages. Au fond, cela revient à réactiver une tradition populaire connue sous le nom de Jaibería, du nom d'un crabe des montagnes (jaiba) se déplaçant latéralement pour avancer. La métaphore de la jaibería renvoie à des pratiques collectives de négociation et à des stratégies de changement qui tout en évitant la confrontation directe s'efforcent d'atteindre les objectifs fixés par des voies détournées...

Ces analyses ont incontestablement le mérite de se défaire de manière radicale des catégories essentialistes qui sont mobilisées par les intellectuels et les acteurs politiques. En déconstruisant les notions de colonialisme et de nationalisme, elles dévoilent une situation dont la complexité ne peut être épuisée par ces catégories discursives. Il est évident que de nouvelles formes d'expression, de "culture " se font jour, à Porto Rico comme ailleurs; que d'anciens clivages se trouvent bousculés et redéfinis et que ces changements doivent être repensés à partir d'une démarche nouvelle. Du même coup, apparait l'inanité de certaines stratégies politiques insulaires, comme en témoigne l'impasse actuelle due au caractère irréconciliable des positions qui s'affrontent dans le champ politique. Ce n'est pas dire pour autant que la déconstruction et la reformulation postmoderniste proposées ici permettront de sortir de l'impasse politique. Significativement, les auteurs se sont abstenus de proposer une traduction politique concrète de leurs prises de position. Car la solution est pour le moins complexe; elle pose un double problème : comment faire pour circonscrire à partir du potentiel de créativité mis en évidence l'espace commun d'une alternative crédible? De ce point de vue, la métaphore de la Jaibería paraît bien insuffisante dans la mesure où elle ne possède pas son propre dépassement. Elle rappelle la stratégie de la ruse telle qu'elle s'est déployée sous l'esclavage et dont la condition de possibilité est précisément l'existence d'un lien de subordination. En l'absence d'un objectif clairement défini fédérant les micro-inventions culturelles, elle peut devenir fonctionnelle par rapport à la domination combattue. D'autant que, et c'est le deuxième problème, les nouvelles formes de lutte et les mouvements qui se recomposent en déplaçant les lignes de clivage traditionnel, peuvent difficilement restés confinés dans leur espace propre en se maintenant à l'écart des grands mouvements traditionnels formés par l'histoire, même si ces derniers ne sauraient être réifiés par l'analyse et doivent se restructurer en termes de stratégie.

Pour José Luis Méndez, ces forces traditionnelles ont pourtant un rôle important à jouer. Son essai intitulé Entre el limbo y el consenso ${ }^{13}$, procède en effet d'un constat simple : à défaut de rallier leurs adversaires à leurs positions, les partis politiques portoricains se contentent d'empêcher que le point de vue de leurs rivaux ne triomphe sur la scène politique insulaire. D'où une sorte d'“alliance pour l'immobilisme " qui perpétue le statu quo et interdit d'opérer la jonction entre les sphères politiques traditionnelles et les forces nouvelles issues de la société civile lesquelles semblent en mesure d'enclencher une dynamique de changement. Dans une langue claire et imagée, José Luis Méndez explique qu'il est possible de sortir de l'impasse actuelle en recherchant une solution démocratique et négociée. A condition toutefois de se dégager de la posture adoptée durant quasiment un siècle; posture consistant à 
enregistrer les désaccords successifs sur la question du statut entre des forces politiques se neutralisant mutuellement.

21 On l'aura sans doute compris, le maître mot de l'ouvrage est le consensus, entendu non pas simplement comme une attitude ou une démarche, mais aussi comme un objectif à atteindre. Un consensus que l'on pourrait envisager à partir de dix points au moins (pp.11-13) parmi lesquels : l'élaboration d'un nouveau modèle économique, l'idée que les Portoricains résidant aux Etats-Unis et ceux vivant sur l'île appartiennent à un seul et même peuple et que les premiers devraient être nécessairement consultés en cas de plébiscite sur le statut...

Cette stratégie fondée sur la recherche d'un accord paraît d'autant plus viable qu'il existe de toute évidence un écart grandissant entre les options politiques incarnées par les trois principaux partis, telles qu'elles se sont cristallisées au cours des années, et les attentes actuelles. Nul doute que le contenu de l'autonomie, de l'annexion et de l'indépendance a profondément évolué, rapprochant partiellement ces différentes options ${ }^{14}$ et libérant un espace possible de négociations. D'autant que par ailleurs, l'examen du contenu de ces options révèle des préoccupations et des aspirations similaires et rappelle, par delà les clivages politiques, l'appartenance à une même culture, la pratique d'une langue commune et l'utilisation des mêmes symboles. Par conséquent, la voie consensuelle est le seul moyen d'éviter une solution imposée depuis la Métropole, comme c'est le cas avec le projet Young - actuellement en discussion au Congrès fédéral - qui n'est que la simple reproduction d'un anachronisme colonial. Pour l'auteur, cette solution passe par une réévaluation des notions qui structurent la vie politique locale, en particulier celle d'annexionnisme et d'assimilationnisme. Ces deux notions sont en effet loin d'être synonymes, même si elles peuvent entretenir un lien étroit et font référence à des comportements qui peuvent se conforter mutuellement: la première désigne une stratégie politique d'intégration institutionnelle; la seconde correspond à un processus de dilution d'une entité culturelle dans un ensemble plus vaste. Après une longue période durant laquelle assimilationnisme et annexionnisme semblaient aller de pair, les années 70 donnent le signal d'un double mouvement divergent: d'un côté l'annexionnisme amorce une ascension ayant permis au Patido Nuevo Progresista (PNP), actuellement au pouvoir, d'alterner régulièrement au gouvernement avec son homologue autonomiste; de l'autre, le déclin du soutien apporté par la population à l'assimilation culturelle et linguistique de Porto Rico aux Etats-Unis. En d'autres termes, on assiste à la montée en puissance d'un annexionnisme sans assimilation qui témoigne de l'échec des tentatives d'américanisation et induit trois conséquences principales: la nécessité pour le mouvement annexionniste de rompre avec un discours à double portée, nationaliste et patriotique lorsqu'il est destiné à l'électeur portoricain, assimilationniste et boiteux lorsqu'il s'adresse aux membres du Congrès ou aux fonctionnaires fédéraux; la place prise par l'espagnol à Porto Rico paraît difficilement compatible avec un projet d'annexion sans assimilation, compte tenu des oppositions très vives dans les milieux conservateurs aux Etats-Unis; enfin, l'annexionnisme devra choisir entre une stratégie à court terme à géométrie variable et ambiguë et une stratégie à long terme clairement assumée et visant à faire accepter par le Congrès fédéral, de manière pleinement consciente, Porto Rico tel qu'il s'est forgé au long de l'histoire.

Plusieurs autres thèmes meublant traditionnellement la vie politique insulaire - la relation entre citoyenneté et nationalité, le problème de la langue, la place du leader 
nationaliste Pedro Albizu Campos dans la société insulaire - sont abordés par l'auteur, sans oublier un chapitre consacré à la salsa analysée ici comme le lieu d'intégration de différentes expériences musicales latino-américaines, un formidable espace de communication et d'échanges se nourrissant, dans le cas de Porto Rico, des flux entre l'île et les Etats-Unis et fortifiant l'identité portoricaine : en adoptant la salsa, Porto Rico s'affirme non seulement comme un pays hispanique ayant des racines afrocaraïbéennes, mais construit simultanément son identité sur le terrain des luttes politiques et sociales aux Etats-Unis en rejetant toute idée d'assimilation dans le cadre du "melting-pot" au sens où l'entendent les secteurs représentatifs de l'hégémonie culturelle anglo-saxonne. Il en résulte que la salsa a servi de rempart à l'assimilation culturelle des classes populaires tout en posant les bases d'une alternative latine, afroantillaise et démocratique face aux prétentions assimilatrices des classes dominantes à Porto Rico.

$24 \mathrm{Au}$ total, José Luis Méndez propose un diagnostic clair et précis de l'impasse portoricaine assorti d'une démarche volontariste susceptible de dégager un espace de négociations entre partenaire/adversaires campés sur des positions jugées irréductibles et une voie nouvelle pour sortir du dilemme dans lequel se trouvent enfermés les Portoricains. Bien sûr, il ne se hasarde pas à envisager des solutions concrètes mais dessine les contours, sinon d'un projet, du moins d'un objectif permettant de réaliser l'intégration, même partielle, de points de vue réputés inconciliables. Encore faudrait-il parvenir à briser l'alliance pour l'immobilisme. Un combat qui est loin d'être gagné, tant il est vrai qu'à Porto Rico les matrices originelles d'alignements partisans tendent à cristalliser les positions, voire à les figer dans un rapport conflictuel parfois éloigné des préoccupations immédiates de la population. Pourtant, il est clair que les idées, elles, évoluent dans la mesure où elles font nécessairement écho aux interrogations du temps et aux nouveaux enjeux qui affleurent la scène sociale et politique. Et s'il est vrai que les partis politiques continuent à structurer très largement la vie partisane, il est non moins certain qu'ils courent le risque sinon de se couper de la population, du moins d'être discrédités comme vecteur d'un changement trop souvent différé.

\section{NOTES}

1. Etat Libre Associé.

2. Il s'agit des Portoricains émigrés aux Etats-Unis.

3. Lors des négociations entamées il y a quelques années sur l'avenir institutionnel de Porto Rico, le Partido Independentista Puertorriqueño (PIP) a plaidé pour le concept de " permanent dual citizenship ".

4. Commonwealth est la traduction anglaise d'Estado Libre Asociado (ELA).

5. Cet auteur écrit très justement: "Le postulat de l'identité comme nécessairement mobile, quand il évacue ou discrimine certains pans de l'expérience sociale, conduit sur le versant d'un autre essentialisme, trait à trait opposé à l'identité "substance". Il n'y a plus assignation identitaire au groupe et au territoire mais assignation identitaire à des formes changeantes et 
hybrides", voir Christine Chivallon, “Du territoire au réseau: comment penser l'identité antillaise ", Cahiers d'Etudes africaines, 148, XXXVII-4, 1997, p. 787.

6. "Puerto Rico : Surviving Colonialism and Nationalism ", pp. 39-56.

7. Voir aussi l'analyse proposée par Yolanda Martinez qui explore les tensions - apparues au sein des femmes portoricaines - liées à des facteurs de classe, de race et aux positions idéologiques (chapitre 6: "Deconsctructing Puerto Ricanness through Sexuality: Female Counternarratives on Puerto Rican Identity (1894-1934) ", pp. 127-139.

8. "The Divorce of Nationalist Discourses from the Puerto Rican People: A Sociohistorical Perspective ", pp. 57-76.

9. "Puerto Rico : The myth of the National Economy", pp. 77-92.

10. Pour sa part Alena Dávila estime que la mondialisation ne signifie nullement l'alignement sur les normes culturelles dominantes. Cet auteur montre comment les firmes américaines implantées à Porto Rico instrumentalisent les représentations culturelles en puisant dans un répertoire - davantage populaire qu'élitiste ou nostalgique - mêlant différentes composantes. (“Rapping Two Versions of the Same Requiem ", pp. 243-256)

11. Agustín Lao, “Islands at the Crossroads : Puerto Ricanness Travelling between the Translocal Nation and the Global City ", pp. 169-188.

12. Frances-Negrón-Muntaner, “English Only Jamás but Spanish Only Cuidado : Language and Nationalism in Contemporary Puerto Rico ”, pp. 257-285.

13. Que l'on pourrait traduire ainsi "Entre la paralysie et le consensus".

14. Un point de vue analogue a été défendu par d'autres auteurs, voir par exemple Angel Israel Rivera \& Aarón Gamaliel Ramos, “The Quest for a New Political Arrangement in Puerto Rico : Issues and Challenges ", Caribbean Studies, 26(3-4), juillet-decembre 1993, pp. 265-292. 